\title{
Clinical and bacteriological profile of neonates admitted in the neonatal care unit of western regional hospital
}

\author{
Shrestha Shree Krishna ${ }^{1}$, Ghimire Jagat Jeevan ${ }^{1}$, Bastola Ramchandra ${ }^{2}$, Gurung Rupa ${ }^{1}$ \\ ${ }^{1}$ Department of Pediatrics, Pokhara Academy of Health Sciences, Western Regional Hospital, \\ ${ }^{2}$ Department of Pediatrics, Matrishishu Miteri Hospital
}

\section{Corresponding Author: \\ Dr. Shree Krishna Shrestha. Chief Consultant Pediatrician, \\ Department of Pediatrics \\ Pokhara Academy of Health Sciences Western Regional Hospital \\ Pokhara, Nepal \\ E-mail : shreekrishnas@gmail.com.}

Article received : December 10, 2017 Article accepted : March 5, 2018

\begin{abstract}
Background : Neonatal sepsis is leading cause of mortality in neonates. The organism responsible for sepsis differed depending on the site of Neonatal care unit and country. In this study, we aimed to look at cause of sepsis and antibiotic sensitivity. Materials and Methods : Neonates admitted in the neonatal care unit of western regional hospital from 14 ${ }^{\text {th }}$ August 2012 to $14^{\text {th }}$ July 2014were included in the study. Septic workup was done and culture was sent. Results of culture sensitivity were noted along with the sensitivity pattern. Results : Neonatal sepsis accounted for 38 percent of total admission in the neonatal unit. Staphylococcus aureus was the most common organism isolated which was followed by Escherichia coli (E coli). Staphylococcus aureus isolated was sensitive to Cloxacillin in $97 \%$ of cases. There were 5 $\%$ mortalities during the study period. Conclusion: Neonatal sepsis was the most common reason for admission and staphylococcus aureus was the most common organism.
\end{abstract}

Keywords: Neonatal sepsis, staphylococcus aureus, mortality, sensitivity

\section{INTRODUCTION}

Neonatal sepsis is the leading cause of mortality in neonates. Sepsis along with prematurity, birth asphyxia and congenital anomalies are leading cause of mortality in neonates. ${ }^{1}$ In 2016, 2.6 million deaths, or roughly $46 \%$ of all under-five deaths, occur during this period. This translates to 7000 newborn deaths every day. ${ }^{1}$ Neonatal sepsis present as wide range of clinical syndrome. Neonatal survivors of sepsis can have severe neurologic sequelae due to central nervous system infection as well as from secondary hypoxemia resulting from septic shock, persistent hypertension, and severe parenchymal lung disease. Refusal to feed along with lethargy represent as the leading presentation of neonatal sepsis. ${ }^{2}$ Due to the changing pattern of antibiotic use and changes in life style there have been change in the spectrum of organism. ${ }^{3}$ Although Group B streptococcus represent major organism for neonatal sepsis, the pattern of organism differs in the developing countries. ${ }^{4}$ Gram negative organism along with staphylococcal infection are responsible for majority of infections in developing countries. ${ }^{5}$ This study was done with objective of understanding the clinical and bacteriological profile of neonates along with pattern of antibiotic sensitivity.

\section{MATERIALS AND METHODS}

Neonates admitted in the Neonatal care unit of Western Regional Hospital from $14^{\text {th }}$ August 2012- $14^{\text {th }}$ July 2014were enrolled in the study. Preceding the study, ethical permission was obtained from the institutional board. Consent from the parents of neonates were also obtained. Patient's history and physical examination was done at the time of examination. Clinical presentation was noted. Septic work was done with CBC (Complete Blood Count), CRP(C-Reactive Protein), micro-ESR ( Erythrocyte Sedimentation Rate) and blood culture to neonates following clinical suspicion of neonatal sepsis. Age of neonate, sex of neonate, diagnosis and final outcome was noted. The report of culture sensitivity was noted along with the pattern of sensitivity. Diagnosis of neonatal sepsis was made with presence of antenatal risk factors along with clinical presentation. Neonatal sepsis 
was suspected with the following criteria:

At time of birth: All newborns born to mothers with maternal fever, or prolonged rupture of membrane $(>18$ hours), or foul smelling or meconium stained liquor and/ or newborn with prematurity $(<37$ weeks of gestational age) or birth asphyxia necessitating active resuscitation

After birth: All neonates with lethargy, refusal to feeds, abdominal distention, respiratory distress, temperature instability (hypothermia/fever), jaundice, seizures, vomiting, and autonomic dysfunction etc.

\section{RESULTS}

There were 1938 neonates during the study period. Out of them 1182 were males and 756 were females.Total1086 presented NNS within 7 days of life and 852 after 7 days of life. Neonatal sepsis was the most common diagnosis .Out of all, 742 were diagnosed with NNS which comprised of $38 \%$ (Table 1 and Table 2). There were 96 mortalities during the study period which comprised $5 \%$. Out of 742 neonates with diagnosis of neonatal sepsis 58 neonates had blood culture positive. (Table 3 )

Table 1: Baseline characters of patients

\begin{tabular}{cccc}
\hline \multicolumn{2}{c}{ Variable } & Number(n) & Percentage (\%) \\
\hline & males & 1182 & 61 \\
Gender & females & 756 & 39 \\
$\begin{array}{c}\text { Age at } \\
\text { pres- } \\
\text { entation }\end{array}$ & $>7$ days & 1086 & 53 \\
Mortality & 852 & 47 \\
\multicolumn{2}{c}{ Culture positive } & 96 & 5 \\
\hline
\end{tabular}

Table 2: Diagnosis of neonates admitted in the ward

\begin{tabular}{lcc}
\hline Diagnosis & $<7$ days & $>7$ days \\
\hline Neonatal sepsis & 532 & 210 \\
Birth asphyxia & 227 & -- \\
MAS & 225 & -- \\
NNJ & 211 & 136 \\
Prematurity & 129 & ---- \\
Staphylococcal & 35 & 28 \\
skin infection & & 106 \\
Pneumonia & 37 & 22 \\
Others & 20 & 502 \\
Total & 1416 & \\
& & \\
\hline
\end{tabular}

\section{Table 3 : Organism isolated from blood culture}

\begin{tabular}{lcc}
\hline Organism & N (58) & percentage \\
\hline Staph aureus & 33 & 56 \\
E coli & 7 & 12 \\
\hline Pseudomonas & 4 & 7 \\
\hline Acinetobacter & 4 & 7 \\
\hline Citrobacterfreundi & 5 & 9 \\
\hline Klebsiella & 2 & 3 \\
\hline Enterobacter & 2 & 3 \\
\hline CONS & 1 & 1.8 \\
\hline
\end{tabular}

Table four below shows the clinical presentation of neonatal sepsis in our study. Majority of the cases showed fast breathing, refusal to feed (18\%)

Table 4 showing the clinical presentation of neonatal $\operatorname{sepsis}(n=54)$

\begin{tabular}{ll}
\hline Clinical presentation & Number $(\%)$ \\
\hline Fast breathing & $10(18.51)$ \\
Fever & $5(9.25)$ \\
Hypothermia & $8(14.81)$ \\
Lethargy & $9(16.66)$ \\
Refusal to feed & $10(18.51)$ \\
Vomiting & $5(9.25)$ \\
Diarrhea & $2(3.70)$ \\
Seizure & $5(9.25)$ \\
\hline
\end{tabular}

Sensitivity pattern of the antibiotics used for the treatment in our study. 
Table 5: Antibiotic sensitivity patterns in common isolates

Name of organism

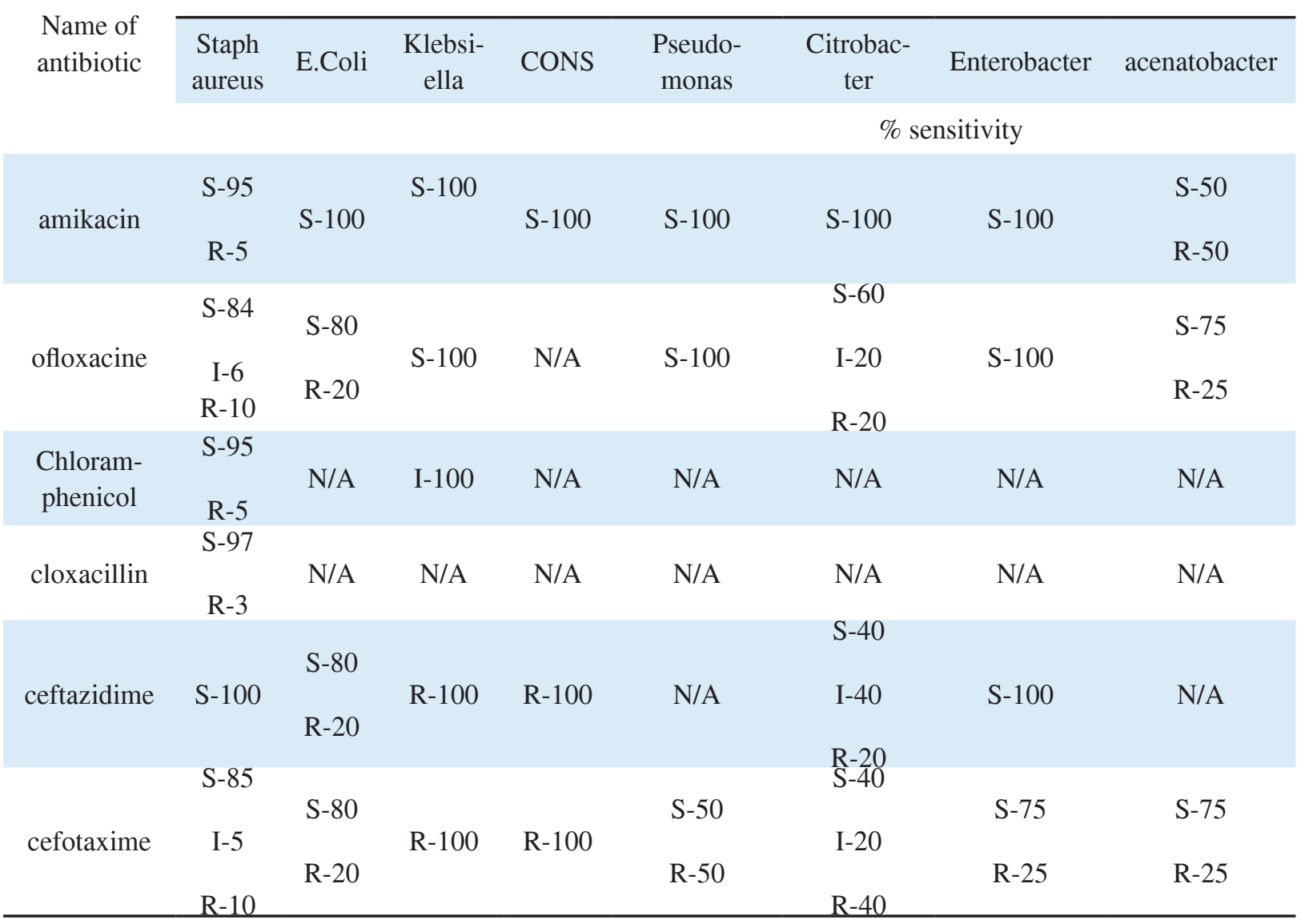

\section{DISCUSSION}

Neonatal sepsis comprised of $38 \%$ of total admissions in our neonatal care unit. This was comparable to the study done by Sridhar et.al. where the percentage of NNS was34\%. ${ }^{6}$ Neonates presented with fast breathing and refusal to feed(18.51\% in each )which was comparable to the study done by Shrestha et al where refusal to feed was most common presentation. ${ }^{5}$ It is comparable to studies done by various authors like Dias et al ${ }^{7}$ Shaw et al ${ }^{8}$, Bhattacharya et al ${ }^{9}$ with $32 \%, 54.64 \%$ and $48 \%$ respectively. This result is comparable to study conducted by Shah et al with $31.57 \%$. presenting as fast breathing and refusal to feed. ${ }^{10}$ Four percentage of cases of neonatal sepsis were positive with blood culture which differed to different studies done in India, Bangladesh and Nepal. ${ }^{5,13}$ This probably resulted from poor lab support for culture in our hospital. Staphylococcus aureus was the most common organism in our study which was comparable to different studies. ${ }^{5,11,12}$ Mortality in our study was $4 \%$ which differed from different studies done in Pakistan, Bangladesh and Nigeria which showed mortalities of $30.9 \%, 20.6 \%$ and $20.3 \%$ respectively. ${ }^{13,14,15}$
This probably resulted from the fact that our neonatal care unit was of level II contrast to the centres of these studies which were level III .

\section{CONCLUSION}

Neonatal sepsis was leading morbidity in the neonatal care unit in western regional hospital. Staphylococcus aureus was most common organism and 3\% of staph aureus were resistant to Cloxacillin.

\section{REFERENCES}

1. WHO.GlobalHealthObservatory(GHO)data.2016. http://www. who.int/gho/child_health/mortality/neonatal_text/en/

2. Anne R. Hansen ECE, Ann R et al.Cloherty and Stark's Manual of Neonatal Care $7^{\text {th }}$ edition. 49: 624-5.

3. Wu JH, Chen CY, Tsao PN et al. Neonatal sepsis: a 6-year analysis in a neonatal care unit in Taiwan.Pediatrics and Neonatology.2009;50(3):88-95.

4. Plazek MM, White LA. Early and late neonatal septicemia. Arch Dis Child. 1983;58:728-31 
5. Shrestha P,D BK, Bhatta NK et al. Clinical and bacteriological profiles of blood culture positive sepsis in newborns. J Nepal Paediatr Soc. 2008;27:64-7.

6. Sridhar PV TP, Sandeep M. Morbidity Pattern and Hospital Outcome of Neonates Admitted in a Tertiary Care Teaching Hospital, Mandya. Int J Sci Stud. 2015;3(6):126-9.

7. Dias E, Viganeshwaran $\mathrm{P}$, The bacterial profile of neoanatal septicemia in a rural hospital in south india, J Clin Diagnosis Res 2010,4:3327-30.

8. Shaw CK. Shaw P. Thapalial A. Neonatal sepsis bacterial isolates and antibiotic susceptibility patterns at a NICU in a tertiary care hospital in western Nepal, A Restrospective analysis. Kathmandu Univ Med J 2008, 5:155-60

9. Bhattecharjee A, Sen MR, Prakash , Gaur A, Anupurba S Increased prevalence of extended spectrum beta lactamase producers in neonatal septicemic cases at tertiary referral hospital. Indian J Med Microbio 2008, 26:356-60.
10. Shah AJ, Mulla SA, Revdiwalla SB, Neonatal Sepsis high antibiotic resiatance of the bacterial pathogens in a neonatal intensive care unit of a tertiary referral hospital. J. Clin. Neonatal 2012, 1(2) $72-5$.

11. Karthikeyan G PK.Neonatal Sepsis: Staphylococcus Aureus As The Predominant Pathogen. Indian J Pediatr. 2001;68:715-7.

12. Thomas M PB, Srimathi G, Sundararajan V et al.Microbial Profile Of Neonatal Infection In Coimbatore. Indian J Pediatr 1999;66: 11-4.

13. Seyal T HF, Anwar A.Audit of neonatal morbidity and mortality at neonatal unit of Sir Gangaram hospital Lahore. Annals of KEMU. 2011 (17(1)):9-13.

14. MN. I. Situation of neonatal health in Bangladesh Orion medical journal 2006; 6:8-10

15. A G , Factors predisposing to low birth weight in Jammu Hospital South Western Ethiopia. East Afr Med J. 2005;82(11):554-8. 